THIRD REPORT ON THEORY OF GROUPS. [Nov.,

\title{
THIRD REPORT ON RECENT PROGRESS IN THE THEORY OF GROUPS OF FINITE ORDER.
}

\author{
BY PROFESSOR G. A. MILLER.
}

(Read before the American Mathematical Society, September 6, 1907.)

THE following general treatises have appeared since the presentation of my second report* a little more than five years ago: The constructive development of group theory with a bibliography, by B. S. Easton, 1902 ; A brief work in Russian entitled The polyhedron groups, by G. W. Pfeiffer, 1903 ; Mathematical crystallography and the theory of groups of movements, by H. Hilton, 1903 ; Eléments de la théorie des groupes abstraits, par J. A. de Séguier, 1904 ; and Quelques considérations sur les groupes d'ordre fini et les groupes finis continus, par Raymond Le Vavasseur, 1904. The number of treatises devoting at least one chapter to our subject has been considerably increased. Two have appeared in America : Dickson, Introduction to the theory of algebraic equations, 1903, and Cajori, An introduction to the modern theory of equations, 1904.

Unfortunately the report by Dr. Steinitz, which was announced by the Deutsche Mathematiker-Vereinigung at different times had to be abandoned. The works by Loewy and Wiman entitled respectively Vorlesungen über die Theorie der linearen Substitutionsgruppen and Endliche Gruppen linearer Transformationen, which were expected to appear in Teubner's Sammlung, have also been delayed. On the other hand Netto is preparing an elementary work for the Sammlung Schubert under the title Gruppen und Substitutionentheorie, and the second part of de Séguier's work is in press.

In view of the very large number of new theorems it appears impracticable to attempt to enumerate all of them. In the case of the larger memoirs where a number of connected theorems are developed I can generally only give such references as indicate the nature of the main questions, while the theorems which have appeared in very brief articles or in less accessible places receive relatively more attention. Moreover, it did not appear best to crowd the report with a complete list

\footnotetext{
* Bulletin, November, 1902, p. 106.
} 
of references, since bibliographical works are more convenient for the purpose of learning all the details along special lines. I hope to have succeeded in exhibiting the connections between some isolated developments which are of importance, and that the report may thus arouse a wider interest along certain lines. It may be added that the advances in the theory of abstract groups have been noted in greater detail than those which come under the other headings of this report.

\section{§ 1. Elementary Applications.}

Among the most elementary and the most important applications of groups of finite order are those which relate to the fundamental operations of arithmetic. The addition and the multiplication groups * were developed principally during the periods covered by the preceding reports. One of the most important extensions of the theory of the latter, made during the period covered by the present report, is given by the following theorem : The necessary and sufficient condition that a given set of numbers, which are distinct with respect to modulus $m$, generates a group with respect to multiplication is that the highest common factor of the modulus and a number of the set is independent of the choice of this number and that the quotient obtained by dividing $m$ by this highest common factor is prime to this factor. $\dagger$

If $x_{1}$ and $n$ represent any two numbers (real or complex), the number $x_{1}-n$ can be obtained geometrically by reflecting the point representing $n$, in the complex number plane, on the middle point of the segment connecting $x_{1}$ with the origin; that is, by connecting the point representing $n$ with this middle point and extending the segment to an equal distance beyond this point. From this standpoint subtraction may clearly be regarded as an operation of period 2 ; and, if $n$ is subtracted from a series of numbers $x_{1}, x_{2}, \cdots, x_{l}$ and the remainders are again subtracted from these numbers, in any order, these operations of period 2 generate a dihedral group and every dihedral group may be generated in this way. It is of interest to observe that the series of numbers $x_{1}, x_{2}, \ldots, x_{l}$ may be replaced by two numbers without affecting this dihedral group, + but a further

p. 77.

* Bulletin, vol. 7 (1900), p. 122; Annals of Mathematics, vol. 2 (1901),

$\dagger$ Annals of Mathematics, vol. 6 (1905), p. 44.

$\ddagger$ Loc. cit., p. 43 . 
reduction is generally impossible. As the group is dependent merely upon the difference of these numbers and the modulus it is clear that every dihedral group may be generated in an infinite number of ways as a subtraction group.

The applications of these groups which have been most fully developed are those connected with the notions of complement and supplement of an angle in elementary trigonometry. As the modulus in this case is $360^{\circ}$, this group is equivalent to the one obtained by subtracting from 1 and 2 with respect to modulus 4. Hence it is identical with the group of movements of the square. The fact that there are two sets of four angles which do not go into eight distinct angles by means of the operations of taking the complement and the supplement is thus related to the general theory that, with respect to the subtraction group, there are always two and only two such sets of numbers, and the total number of different numbers in the two sets is equal to the order of the dihedral group. The more general applications of the subtraction groups in elementary trigonometry have also been considered and a few illustrative examples are given in a recent article in the Annals of Mathematics.*

Results of unusual interest relate to the groups generated by the two operations of subtracting from a fixed number and dividing either the same or a different number. These groups differ from those considered above with respect to the fundamental property that it is not necessary to assume a modulus in order to obtain finite orders. If the subtrahend is denoted by $x_{1}$ and the dividend by $x_{2}$, the two operations $x_{1}-n, x_{2} / n$ generate a dihedral group, since each of these operations is of order 2. The important result that only four distinct groups of finite order are possible when $x_{1}$ and $x_{2}$ are both rational numbers has been proved both analytically and geometrically. $\dagger$ The orders of these groups are 4, 6, 8, and 12 . These orders are obtained by assuming $x_{1}=0, x_{1}^{2}=x_{2}, x_{1}^{2}=2 x_{2}$, and $x_{1}^{2}=3 x_{2}$ respectively. There are three sets of conjugate numbers under such a group which include all the numbers which are transformed into a smaller number of distinct values than the order of the group.

* Vol. 8 (1907), p. 97.

$\dagger$ Quar. Jour. of Mathematics, vol. 37 (1905), p. 80; Hilton, Messenger of Mathematics, vol. 35 (1905), p. 117.

$\ddagger$ Quar. Jour. of Mathematics, loc. cit. 
If it is not assumed that $x_{1}, x_{2}$ are rational, it is possible to assign them values so that the two operations $x_{1}-n, x_{2} / n$ generate a dihedral group of any given possible order. One set of values which leads to the dihedral group of order 10 is $x_{1}=1+\sqrt{ } 5, x_{2}=4$. Among the sets which give rise to the symmetric group of order 6 , that in which $x_{1}=x_{2}=1$ has received most attention, as this is the group formed by the six values of an anharmonic ratio as well as by the six trigonometric functions $\sin ^{2} \alpha, \sec ^{2} \alpha,-\cot ^{2} \alpha,-\tan ^{2} \alpha, \csc ^{2} \alpha$, $\cos ^{2} \alpha$. As the four-group is the only invariant subgroup (besides identity) of a symmetric group which gives rise to a quotient group whose order exceeds 2, the six values of an anharmonic ratio furnish a unique instance of a more than two valued rational function all of whose values are rationally expressible in terms of one of them. That is, any other function having this property is rationally expressible in terms of an anharmonic ratio.

Another instance of very elementary applications which were developed during the period covered by the present report is furnished by the groups of the figures of elementary geometry.* The group of a figure is composed of all the movements of space which transform the figure into itself. In these movements space is regarded as rigid in the sense that any two points are transformed into two points at the same distance from each other. Every movement which transforms a system of points into itself must transform the center of their minimum circumscribing sphere into itself. Hence such movements must be composed of rotations around this center, and all the groups of movements of a system of coplanar points, finite in number, must be either cyclic or of the dihedral rotation type. Moreover, the dihedral group of order $2 n$ is the group of the regular plane polygon of $n$ sides. In particular, the octic group is the group of movements of the square; while the rectangle with unequal sides has the transitive four-group for its group of movements, and the rhombus which is not a square has the intransitive four-group for its group of movements. If the base of a regular pyramid has $n>3$ sides, its group is cyclic and of order $n$, and if the base of a regular prism has $n$ sides $(n \neq 4)$, its group is dihedral rotation and of order $2 n$.

During the last few years some of the direct applications of

*Amer. Math. Monthly, vol. 10 (1903), p. 215. 
substitution groups in elementary trigonometry have received notice.* If $a, b, c$ represent the sides of a spherical triangle, and $\alpha, \beta, \gamma$ the supplements of the angles opposite those sides in order, it follows from the properties of the polar triangle that the substitution $a \alpha \cdot b \beta \cdot c \gamma$ transforms any formula relating to the spherical triangle into one which is equally true. It is also evident that any formula which has been derived without specializing any one of the six parts in question is transformed by the two substitutions $a b \cdot \alpha \beta, b c \cdot \beta \gamma$ into one which is equally true. Hence it follows that the group of order 12 which is generated by these three substitutions transforms any such formula into those which are equally true. This well known imprimitive group of order 12 and degree 6 may therefore be employed to derive a system of formulas from a given one, and hence it may sometimes be used to advantage even in a very elementary course of instruction. Its intransitive subgroup of order 6 may be employed to some advantage in plane trigonometry.

\section{§ 2. Abstract Groups.}

During the period under consideration great progress was made in the theory of abstract groups, just as had been the case during the preceding period. I will first state some of the new theorems relating to generational operators. If a series of operators is such that each of them transforms all the others either into their inverses or into their third powers, the totality of these operators generates the hamiltonian group of order $2^{n}$, except when all of them are of order $2 . \dagger$ In this special case it generates the abelian group of order $2^{m}$ and of type $(1,1,1, \ldots)$. If a non-abelian group is generated by two operators which transform each other into their $\alpha, \beta$ powers respectively, the group is completely defined by these powers whenever the indices of the powers diminished by one are such that one of them is a prime while the other is a power of this prime, and only then. In other words, the non-abelian group of order $p^{m}$ which contains a cyclic subgroup of order

\footnotetext{
* Quar. Jour. of Mathematics, vol. 37 (1906), p. 226.

$\dagger$ From this theorem we may readily derive two very simple definitions of the quaternion group, viz., the quaternion group is the only non-abelian group generated by two operators which transform each other into their third powers, and the quaternion group is the only non-abelian group generated by two operators which transform each other into their inverses. Prace Matematyczno-fizyczne, vol. 17 (1906), p. 120.
} 
$p^{m-1}$ is the only group, besides the quaternion, which is completely defined by the facts that it is non-abelian and that it generated by two operators which transform each other into given powers. Any series of operators such that each is transformed into a power of itself by all the others generates an abelian or a metabelian group which is therefore the direct product of its Sylow subgroups, but not every metabelian group can be generated in this way.*

If three operators are such that the product of any two is equal to the third, they generate either a dihedral group or the quaternion group. $\dagger$ If $n$ different operators are commutative and if each of them is the product of all the others, they generate the direct product of a cyclic group whose order divides $2(n-2)$ and an abelian group of order $2^{a}$ and of type $(1,1,1, \ldots)$. Moreover, any such direct product may be generated by $n$ operators which satisfy the given conditions. If the order of one of these operators is divisible by 4 , all of them are of the same order. Every possible symmetric group whose degree exceeds 3 may be generated by five non-commutative operators such that each of them is the continued product of the other four, arranged in a given order. The groups generated by four operators such that each is the product of the other three, arranged in a given order, are similar to the dihedral type and have been completely determined. $\neq$

It is well known that the groups of genus zero exhibit the most interesting generational relations. All of these have recently been generalized by replacing two of the three ordinary equations which define such a group by a single one while the third is maintained in the usual form. A large number of new and comparatively simple definitions of groups are thus obtained. $\S$ Closely related to the groups of genus zero are those which may be defined by the orders of two operators and the order of their commutator. When two operators of order 2 have a commutator of an even order, they generate a group whose order is four times the order of this commutator; when they have a commutator of odd order, they generate a group whose order is either twice or four times the order of their commutator. If an operator of order 2 and an operator of order 3

*Quar. Jour. of Mathematics, vol. 37 (1906), p. 286.

$\dagger$ Bulletin, vol. 13 (1907), p. 382.

$\ddagger$ Bulletin, vol. 13 (1907), p. 433 .

\$ Transactions Amer. Math. Society, vol. 8 (1907), p. 1. 
have a commutator of order 2 , they generate either the tetrahedral group or the direct product of this group and the group of order 2. If two operators of order 3 have a commutator of order 2, they generate a group whose order is one of the four numbers $12,36,144,288$.*

Netto recently published a very elementary article on the construction of abstract groups which are generated by two operators, $\uparrow$ in which he obtains a number of well-known results by the use of the most elementary principles and also arrives at a few interesting new relations. It is to be regretted that he failed to indicate where his work has contact with older publications. For instance, the conditions $a^{3}=1, b^{3}=1, b a=a^{2} b^{2}$ on page 255 of Netto's article are clearly equivalent to the well known abstract definition of the tetrahedral group, since $(b a)^{2}=$ $1, b a$ being equal to its inverse ; yet nothing whatever is stated about this and the reader who is not familiar with the subject would naturally infer that the results were due to Netto. The last section of the article is devoted to the proof that the group generated by two operators of order 4 whose product is of order 2 has an infinite number of distinct operators. This is a very special case of an older general theorem in regard to the groups that may be generated by two operators when only their orders and the order of their product are given.

In volume 9 of the Bullers,, Dickson gave useful generational relations for the simple groups of orders 360,504 , and 660, and in volume 35 of the Proceedings of the London Mathematical Society he gave such relations for an abstract simple group of order 4080 as well as for the more general abstract group which is simply isomorphic with the linear fractional group in $G F\left[2^{n}\right]$. In a later volume of the latter journal Bussey gave the generational relations for an abstract group simply isomorphic with $L F\left[2 p^{n}\right] . \S$ Le Vavasseur, Potron, and Neikirk have given a very large number of generational relations as regards the groups of order $p^{m} . \|$

One of the most fundamental recent theorems relating to abstract groups is due to Frobenius. It affirms that the num-

* Bulletin, vol. 13 (1907), p. 371. Cf. Proc. Amer. Philosophical Society, vol. 46 (1907), p. 146.

† Netto, Crelle, 128 (1905), p. 243.

$\ddagger$ Amer. Jour. of Mathematics, vol. 24 (1902), p. 96.

\& Bussey, Proc. London Math. Society, vol. 3 (1905), p. 296.

|l Le Vavasseur, loc. cit., p. 26 ; Potron, Thèse, Paris, 1904 ; Neikirk, thesis, Publications of the University of Pennsylvania. 
ber of the operators whose $n$th power is equal to a given operator $A$ is divisible by the highest common factor of $n$ and $g$, where $g$ represents the number of the operators of the group which are commutative with $A$.* $^{*}$ Another recent fundamental theorem relates to the form of the total number of cyclic groups of order $p^{m}(m>1)$ and asserts that the number of cyclic subgroups of order $p^{m}$ in any group $G$ is always of the form $k p$ whenever the Sylow subgroups of order $p^{a}$ in $G$ are non-cyclic, and that the number of subgroups of order $p$ in such a $G$ is of the form $1+p+k p^{2}, p$ being an odd prime number. $\dagger$ If a group of order $2^{m}$ contains an odd number of cyclic subgroups of order $2^{a}(\alpha>2)$ this number must be unity and the group of order $2^{m}$ contains a cyclic subgroup of order $2^{m-1}$. Moreover the number of operators of order 2 in any group of order $2^{m}$ which does not involve an operator of order $2^{m-1}$ is always of the form $4 k+3, k$ being a positive integer. . If a group of order $2^{m}$ contains exactly two cyclic subgroups of order $2^{m-1}$, the number of the operators of order 2 is also of the form $4 k+3$; but when it contains only one such cyclic subgroup, this number is of the form $4 k+1$. If a Sylow subgroup of order $p^{a}$ in $G$ is cyclic, the number of the subgroups of order $p^{m}$ is evidently of the form $1+k p$.

The study of the groups whose subgroups possess given properties has led to interesting results. From the properties of the non-abelian groups in which every subgroup is abelian, Dickson deduced the form of the order of groups which are necessarily abelian. $\$$ This result is closely related to properties of the orders of groups which are necessarily cyclic. These properties were stated in my first report, page 235 . The results as regards the non-abelian groups in which every subgroup is abelian have been extended by the determination of all the non-abelian groups in which every subgroup is either abelian or hamiltonian, as well as all those in which every subgroup is either abelian or dihedral.\| These conditions led to remarkable elementary categories of groups which will probably continue to be useful, in view of their simple definitions.

All of the groups which were considered in the preceding

\footnotetext{
* Frobenius, Berliner Sitzungsberichte, 1903, p. 987.

$\dagger$ Proc. London Math. Society, vol. 2 (1904), p. 142.

† Transactions Amer. Math. Society, vol. 6 (1905), p. 58.

3. Dickson, Transactions Amer. Math. Society, vol. 6 (1905), p. 201.

|| Transactions Amer. Math. Society, vol. 8 (1907), p. 1; Amer. Jour. of Mathematics, vol. 29 (1907), p. 289.
} 
paragraph are solvable. This is also true of the category which satisfy the condition that any two conjugate operators are commutative, as well as of the historically more interesting category composed of the groups whose orders are of the form $p^{a} q^{\beta}, p$ and $q$ being prime numbers.* Special cases of such groups had been proved solvable by Frobenius, Jordan, Cole, and others before Burnside succeeded in establishing this property for the entire category. This seems the most interesting result as regards solvability which was reached during the period under consideration.

Bachmann has observed that the theory of congruences and Fermat's theorem are based upon the "fundamental mathematical concept of groups" and he employed the elements of this subject in his classic work on number theory. A large number of relations between number theory and group theory have recently been considered. $\dagger$ In many instances the known theorems of these subjects express the same fundamental facts in different languages. This is the case as regards the generalized Wilson's theorem and the theorem giving the continued product of all the operators of an abelian group, $\$$ which was first stated by Zsigmondy. As Zsigmondy's definition of a group is not in accord with later usage, some of the results cannot be directly employed and hence his valuable memoir has not received the notice which it deserves. For instance, it gives a correct statement of the possible types of subgroups of abelian groups, $\S$ while Burnside in his later Theory of Groups came to erroneous conclusions, as has been pointed out once more by Hilton in his recent article in the Proceedings of the London Mathematical Society, volume 5 (1907), page 1. A number of other known results are proved in this article without giving due references.

If a group contains one invariant subgroup of index $p$, the number of the invariant subgroups of this index is of the form $1+p+p^{2}+\cdots+p^{\lambda}, p$ being any prime number. In particular, the number of subgroups of half the order of any group is some number of the series $0,1,3,7,15, \ldots$. The value of $\lambda$ in the preceding expression cannot exceed $m-1$ when $p^{m}$ is the order of a Sylow subgroup of a group. If $p=2$ and

* Burnside, Proc. London Math. Society, vol. 1 (1904), p. 388 ; also, vol. 35 (1903), p. 28.

† Amer. Jour. of Mathematics, vol. 27 (1905), p. 315.

Fite, Annals of Mathematics, vol. 6 (1904), p. 7.

\& Zsigmondy, Monatshefte für Math. und Physik, vol. 7, (1896) p. 205 
$m=1, \lambda$ must have this maximal value. The possible groups of order $p^{m}$ when $\lambda=m-2$ have been considered under the heading "Generalization of the hamiltonian groups" and the analogies between these groups and the hamiltonian groups have been studied. For smaller values of $\lambda$ the difficulty of the problem of determining all the possible groups greatly increases, so that the case when $\lambda=m-3$ has not yet been completely solved.* Another generalization of the hamiltonian groups is due to Wendt and was published in the Mathematische Annalen almost simultaneously with the appearance of a paper dealing with the same question, in a somewhat more restricted manner, and bearing the title "The groups in which every subgroup of composite order is invariant." + The number of the possible invariant subgroups of index $p^{2}$ has also been studied but the results are less simple than those in regard to invariant subgronps of index p.\$ Several interesting general theorems have been deduced from the one which affirms that the number of cyclic subgroups of order $p^{m}$ in any group whose Sylow subgroups of order $p^{\alpha}$ are non-cyclic is divisible by $p$ whenever $p>2$ and $m>1$. One of these relates to the number of cyclic subgroups of any order $k=p_{1}^{a_{1}} p_{2}^{\alpha_{2}} \cdots p_{\lambda}^{\alpha_{\lambda}}, p_{1}<p_{2}<\cdots<p_{\lambda} ; p_{1}, p_{2}, \cdots, p_{\lambda}$ being distinct prime numbers. If any group contains more than one cyclic subgroup of order $k$, it contains at least $p_{1}$ such subgroups, and if it contains exactly $p_{1}$ such subgroups, then $a_{1}>1$ and it contains only one subgroup of order $p_{\lambda}^{a_{\lambda}}$ $(\lambda>1)$. In particular, there is no group which contains exactly two cyclic subgroups of the same order when this order is either odd or twice an odd number while there is an infinite number of different groups which contain exactly two cyclic subgroups whose order is an arbitrary number divisible by 4 . A necessary and sufficient condition that a group is the direct product of its Sylow subgroups is that the $n$th power of each of its operators is contained in every subgroup of index $n$, for every possible value of $n . \S$

Characteristic subgroups have the same property with respect to transformations under the group of isomorphisms as invariant subgroups have as regards transformations under the

* Transactions Amer. Math. Society, vol. 6 (1905), p. 326.

† Bulletin, vol. 12 (1906), p. 379; Archiv der Math. und Physik, vol. 11 (1906), p. 76.

$\ddagger$ Comptes rendus, vol. 140 (1905), p. 32.

\& Amer. Math. Monthly, vol. 13 (1906), p. 10. 
group. As the group of isomorphisms is continually receiving more attention, so the characteristic subgroups are continually playing a more prominent rôle. The groups in which every subgroup is characteristic are cyclic, but all others with the exception of the elementary groups, that is, those which are either simple or the direct product of simply isomorphic simple groups, have both characteristic and non-characteristic subgroups. The most important characteristic subgroups are generated by the commutators of the groups which are not perfect. All the characteristic subgroups of an abelian group have been determined and it has been proved that they have a common subgroup known as the fundamental characteristic subgroup. The subgroup generated by the $i$ th commutators is also characteristic.*

The characteristic subgroup generated by all the invariant operators of a group corresponds to the identity of the group of cogredient isomorphisms and hence it is of such unusual importance as to have received special names. In view of its dependence on this quotient group, it was first called the cogredient subgroup, $\dagger$ but in the text-book of de Séguier and in other French literature it has been denoted by the briefer term the central, while the corresponding quotient group has been called the cogredient. This quotient group is abelian in an important category of groups studied by Fite and others and known as metabelian groups. :

The theorem that every group of order $p^{m}$ contains an abelian subgroup of order $p^{a}$ whenever $m>\frac{1}{2} a(a-1)$ has been extended by showing that the number of these abelian subgroups is of the form $1+k p$ and hence at least one of them is invariant, and also by proving that every group of order 64 contains an abelian subgroup of order 16, while there are groups of order $p^{6}$ for all odd values of $p$ which do not contain an abelian subgroup of order $p^{4}$. In the Decennial Publications of the University of Chicago Moore determines all the subgroups of the generalized finite modular group. As noted in the preceding report, Wiman investigated the same problem, but Moore had practically completed his investigations before the appearance of Wiman's memoir.\| The groups in which every two conjugate operators are commutative and those in

* Fite, Transactions Amer. Math. Society, vol. 7 (1906), p. 61.

†Amer. Math. Monthly, vol. 5 (1898), p. 221.

Fite, Transactions Amer. Math. Society, vol. 7 (1902), p. 331.

\& Messenger of Mathematics, vol. 36 (1907), p. 188.

\| Cf. Jahrbuch über die Fortschritte der Mathematik, vol. 34 (1905),p. 172. 
which every pair of commutative operators, apart from identity, are conjugate have been investigated by Burnside and Rietz respectively.* It was found that the symmetric group of order 6 is the only group which satisfies the latter condition.

In volume 6 of the Transactions of this Society, Professors Moore, Dickson and Huntington extended their earlier results in regard to the definitions of an abstract group. Moore proves that one of the postulates in his earlier definition is redundant and establishes the mutual independence of the postulates in the new definition. Huntington gives a brief historical sketch of abstract definitions of groups and fields, and states that the first abstract definitions of fields are due to Dickson and himself, and are natural extensions of the sets of independent postulates which had already been given for groups. In various memoirs Dickson has developed a theory of groups in an arbitrary field defined by finite groups and has shown, in particular, that when the field is the general Galois field of order $p^{n}$ there is a doubly infinite system of finite groups which corresponds to each given finite group. $\dagger$ The work has close contact with the two earlier papers of Burnside, "On the continuous group that is defined by any given group of finite order."

If the number of operators of order 2 in an abelian group is at least equal to half the order of the group, then the group contains no operator whose order exceeds 2. There are, however, many different types of non-abelian groups in which the number of operators of order 2 is more than half the order of the group. The number of operators whose orders exceed 2 in such a group cannot be less than one fourth of the order of the group, and if it exceeds this number it must be at least equal to one third of the order of the group. For the groups of order $2^{n}$ all the possible ratios between the number of the operators whose orders exceed 2 and the order of the group have been determined on the hypothesis that this ratio is less than one half, and the maximum number of operators of order 2 in a group of any given order has been found. $\$$

In the enumeration of all the possible groups which satisfy given conditions encouraging progress has been made. As noted in my preceding report, the groups whose orders are the products

* Rietz, Transactions Amer. Math. Society, vol. 5 (1904), p. 500.

† Dickson, University of Chicago Decennial Publications, vol. 9 (1902), p. 35 ; Transactions Amer. Math. Society, vol. 3 (1902), p. 285.

† Bulletin, vol. 13 (1907), p. 235. 
of four prime numbers had all been considered with the exception of the case when the order is of the form $p^{2} q r$. The possible groups whose orders are of this form have recently been enumerated by Glenn.* The most extensive enumeration which has appeared during the period covered by the present report is that given in Potron's Paris University thesis. The author of this work aims to give a complete enumeration of all the possible groups of order $p^{6}$ together with some general types of groups of order $p^{m}$. Among the latter are those in which every subgroup of order $p^{m-2}$ is abelian while the group itself is metabelian. In a later note the author removes the latter restriction and makes some corrections of his enumeration of the groups of order $p^{6} . \dagger \mathrm{He}$ uses the term commutant instead of the older terms derivative, or commutator subgroup.

Another extensive enumeration is due to Neikirk and is devoted to the group of order $p^{m}(p>2)$ which involve cyclic subgroups of order $p^{m-3}$. $\$$ The special case where $p=2$ has been considered more recently by Miss McKelden.§ Among the less extensive enumerations which are not mentioned in connection with other subjects in the present report are the following: The groups which contain less than 15 operators of order 2 and are generated by these operators; $\|$ the groups of order $p^{m}$ which contain exactly $p$ cyclic subgroups of order $p^{a}$, those of order $2^{m}$ which contain an odd number of cyclic subgroups of composite order, and those which contain only three operators which are squares of other operators in the group.

All the operators of a gronp of composite order are contained in its cyclic subgroups but it is not always possible to select these subgroups in such a way that every two have only identity in common. The more general question of finding the properties of a group which satisfies the condition that all its operators are contained in a series of subgroups such that any two have only identity in common has been partially studied. The most interesting theorems that have been established may be stated as follows: If the group is non-abelian and of order $p^{m}$, then only one of these subgroups can involve operators of

* Glenn, Transactions Amer. Math. Society, vol. 7 (1906), p. 137. 300.

† Potron, Bulletin de la Société math. de France, vol. 32 (1904), pp. 296,

$\ddagger$ Neikirk, Publications of the University of Pennsylvania, No. 3, 1905.

$\S$ Amer. Math. Monthly, vol. 13 (1906), p. 121.

II Amer. Jour. of Mathematics, vol. 29 (1907), p. 1.

T Transactions Amer. Math. Society, vol. 7 (1906), pp. 94 and 238; vol. 6 (1905), p. 58. 
order $p^{2}$. If it is abelian, its order must be of the form $p^{m}$ and its type must be $(1,1,1, \ldots)$.*

Dickson has found a new system of simple groups by the study for modulus 2 of a linear group on 7 variables which he had investigated before for fields not having this modulus. The problem for modulus 2 required a different analysis and led to a simple group of order $2^{6 q}\left(2^{6 q}-1\right)\left(2^{2 q}-1\right), q>1$. For $q=1$ the group has a simple subgroup of index 2 and of order 6048. $\dagger$ The following theorem is frequently useful in the study of simple groups, especially if they are represented as substitution groups. If $p^{\alpha}$ is the highest power of the prime $p$ which divides the order of a simple group $K$ and if $K$ contains less than $(p+1)^{2}$ subgroups of order $p^{a}$, then each of these Sylow subgroups is transformed into itself by a maximal subgroup of $K$.

In view of the fundamental importance of Sylow's theorem we add the following new statement of it, even though this statement can be readily derived from the one usually given. If the order of a group $G$ is $p^{\alpha} m, p$ being any prime which does not divide $m, G$ contains at least one subgroup $P_{a}$ of order $p^{a}$. Moreover if $P_{\alpha}$ contains only one subgroup $P_{\beta}$ of a given type, then all the subgroups of this type are conjugate under $G$ and their number is of the form $1+k p$. The order of $G$ may be written in the form $p^{\beta} n(1+k p), p^{\beta} n$ being the order of the largest subgroup which transforms $P_{\beta}$ into itself. $\S \quad$ By letting $\beta=\alpha$ we obtain an ordinary form of this theorem.

(To be continued.)

\section{NO'TES.}

The concluding (October) number of volume 29 of the American Journal of Mathematics contains the following papers : "On twisted quintic curves," by E. C. ColpitTs ; "Attraction of the homogeneous spherical segment," by G. W. HILL; "On a certain class of algebraic translation surfaces," by J. EIESLAND; "Group characters of various types of linear groups," by H. E. JoRDAN.

\footnotetext{
* Bulletin, vol. 12 (1906), p. 446.

† Dickson, Mathematische Annalen, vol. 60 (1905), p. 137.

$\ddagger$ Comptes rendus, vol. 136 (1903), p. 294.

$\&$ Annals of Mathematics, vol. 5 (1904), p. 187.
} 